$\begin{array}{cc}\text { 1) ramile } & \text { Sechon } \\ \text { en } \mathrm{mmin} & \text { en } \mathrm{mm}= \\ 0,8 & 0,5 \\ 0,9 & 0,65\end{array}$

$0,9 \quad 0,65$

23

3

lils pour appareillage des lustres ;

isolés sous tubes ou sur isolateurs dont la dislance est mférieure à $1 \mathrm{~m}$. 30 ; conducteurs nus à l'intérieur des bàlıments ;

conducteurs isolés (à l'mtéreur ou à liextér'leur) dont les portées dépassent $1^{\mathrm{m}} 50$ sans être supérieules à 5 mètres.

$2,5 \quad 5 \quad$ conducleurs nus (curvre dur) pour lignes aéruennes de basse el moyenne tension; conducleurs nus à lintérieur des bâtıments pour haute tension;

cábles sous plomb avec armature de fer feullard;

37 conducteur's nus (ouivre dur) pour lignes aériennes de haute tension jusqu'à 5000 volts, quand les portées restent inférieures ì 30 mètres ;

conducleums pour lignes cie terre;

3,59 conducteurs nus (curve dur) pour lignes aéruennes de haute tension au-dessus de 5.000 volts, ou quand les portées dépassent 30 mètres.

b) Les conducteurs nus servant à la construction des lignes aériennes deviont, en gélléral, etre en curvie dur, ou, s'us ont une résistance spécifique à la rupture dufférente de celle du curvre dur (vor $\$ 18-a$ ), ils devront avor des dimensions telles que leur résistance à la rupture égale au moms celle que comporteratent, dans chaque cas, les dmensions minma correspondanles indiquées plus haut, en prenant pour base une résistance à la rupture de $35 \mathrm{kllogr}$. par millimètre carré.

1). - Matériel de pose. isolateurs, tubes, mollures, crampons

$\$ 30$. - Isolateurs. - a) On entendra par isolateurs les suppoits directs des conducteurs, qui devront elre en matiere isolante, incombustıble, maltérable et insensible à l'humidilé. Leur forme devra ctre telle quils ne pussent endommager les conducteur's ou leur isolant.

b) Les isolateurs peuvent en général rentrer dans les catógories survantes :

10 Isolateurs à cloches muliples, - Les isolateurs à cloches multiples pourront etre employés dans tous les cas tant à l'extéremr qu'd lintérkur des batments, a la condition d'ètre appropriées à la tension. Ils devront être placés de manière à ne pas permettre laccumulation de leau dans les cloches.

Les isolateur's qui n'auront qu'une double cloche ne pourront etre employés à liextérieur et dams les locaux moullés que sils ont au moins 80 millim. de hauteur pour les tensions supérıeures à 300 volts et $60 \mathrm{milhm}$. pour les tensions inférieures.

$2^{\circ}$ Isolaleurs à simple cioche. - Les usolateurs à simple cloche ne pourront etre employés a l'extérieur ou dans les locaux moullés que jusqu'à 300 volts et à condition davorr une hauteur de 80 millm. au moms. Ils devront également etre posés de manière à ne pas permettre l'accumulation de l'eau dans la cloche.

$3^{\circ}$ Poulzes à nervures et poulzes-cloches. - Ces isolateurs ne pourront être employés à l'extérieur que s'ils ne sont pas exposés it la pluse. Leurs dimensions devront elre en rapport avec lhumsdité du local, la tension et l'isolation des conducteurs avec un minumum de 35 millim. de distance sumerficielle de la gorge à la base ct a la tetic de vis.

Il est recommandable de grasser les vis de fixation dans les locaux humides.

$1^{\circ}$ Poulues hautes. - Les poulies hautes, c'est-it-dre celles avant au moins une embase de 10 millim. de haulcur, peuvent cire employées dans les memes condilions que les pricédentes lanl que l'humidité des locanx le permet.

$5^{\circ}$ Poulles basses. - Les poulles basses fixes conire les pamos ne peuvent etre cmployées que dans les locaur secs et jusqua à 600 volts.

$6^{\circ}$ Taquets - I.es taquets pourront etre employés dans les memes condilions que les poulies survant la hauteur de leur embase.

$\$ 31$. - Tubes. - a) Les tubes ne devront pas présenter a l'intérieur d'arête vive pouvant endommager l'isolation du conducteur pendant la pose.

b) Les tubes isolants devront etre constutues par une matière inaltérable it l'air et à l'humidité. Ils pourmont, d'ailleurs, avoir une armalure nédallque qui sera de rigncur a parta de woo volts (voir $(l)$.

c) Les lubes métalliques non gamis inténeurement a une matiere solante devont elre condus moxydables el en geniral msensibles a laction chinnque du mileu ambianl. Is ne devont contenir que des fils ayant au moms une isolition fonte (:30) megohms).

d) Les lubes métalliques, ansi que l'enveloppe milallupe do tubes isolants, devront conshluer, s'll $y$ a lien, une protection efficace pour les conductems, en parlinulier, sils sont destmés ¿t etre logés dans les murs, jls devront arour une résistance mí canque suffisante pour empecher la pénétration le clous ou do villes.

Jour la moyenne et la haule tension, los racorts derront ctre reliés ćlectriquement et l'armature mise a la terre.

e) Lorsquion se sent de tubes a armature molallipue pour $y$ loger des conducleurs de courants allematifs, $11 \mathrm{v}$ a lieu in genéral de loger les fils d'aller et de rotour dans le mome tube poux éviter l'échaulfement de l'amature par sulte des comanls dinduction.

f) I.e dramètre intémeur des tubes, le nombre des coucies el leur rayon annsi que le nombre des bolles de jonction dorvent etre chorsis de telle manuère qu'on puusse en foul tenjus panser ou retrer des conducteurs. Les branchements, durvations of raccords des conducleuris ne dorvent pas se fame dans les luyaux memes, mas dans des boîtes de foncilon fu om pusse ouvmu facilement en tout temps.

g) Liemplor de tubes contenant plusicurs fils sera restroint anc locaux absolument secs, à des tensions nitiricures a boo volts. Les conducteuns devront avour au moms une solutum forle (30n) mégohms). Un mome luyau ne dost nas coutenn, en ginólal, plus de trois conducleurs.

h) Les tuyaux contenant des conducteurs doivent elre disposés de fason à empecher l'entrée et l'accumulation de leau.

$\$ 32 .-$ Moulures. - a) Les moulures dorvent efre en bols sec ; lintervalle entre les ramunes doul avom au moms 6 mmllm. La ligne de pose des pointes dont ctre indiqués sin te convercle.

b) Les moulures ne peuvent elre employées que dins les lomax parfartement secs, à des tensions mfóloures à 600 volls, of 110 doivent contenir que des fils ayant au moms une isolation forle (300 mógohms). Elles ne pourront dre placeses en dossous dis conduites d'eau ou de vapeur sans dre cffeacement proligries contre la chute des gonttes deau. Leur écartement minimum des conduites et pieces métalliques sera re 30 mullmolses

c) Les moulures dorvent etre endutes avanl lem jose, et an moins sur la face regardant le mur, dun produt cmorchant

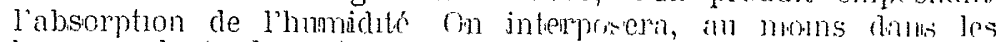
locaux mdustruels, entre les muns of les momlules, les cales, de manière a laisseł derrière les moulures un espace darr de 3 nillimètres.

d) Les fils seront posés librement dans les raimuros a rason d'un fil par ramure et sans dire mainkenus par des pointes.

e) Les moulures devront tomjours rester apparentas, c'est-i-dire ne pas ctre recouvertes de paprer ou de tentures, el encore moms de crépissage.

f) Si plus de deux moulures sont poseres mamllinment, il sera bon d'apposer d'une maniere visible des marques pour pertnellete de suivre les dilférentis circuits.

\$ 39. - Crampons. - Les crampons ne pourponl ptre employes

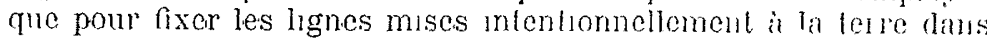
les endroits sces.

Leur emploi ne devra pas provoquer de deterioration des conducteurs.

(Asulve)

\section{AMORTISSEUR DE COUPS DE BÉLIER à l'Usine hydro-électrique de Tumwater Ganyon}

La compagnie du Great Northern Railway ayant decide lélectrification du tunnel de la Caccade, dans l'élat do Washington, sur sa ligne transcontinentale do Scattle, sur le Puget Sound, à Chicago, par Spokane, a fait aménager une chute de la Wenachee River, à Tumwater Canyoll.

L'eau est amenée à l'usine au moyen d'une conduite forcéc 
de $2{ }^{m} 59$ de diamètre, longue de $3,6 \mathrm{kms}$ (dont 3,3 en bois). L'usine génératıice comporte 2 groupes électrogènes, composés de 2 turbines de $4000 \mathrm{H}$. P. fonctionnant sous $60 \mathrm{~m}$. de chute et actionnant 2 alternateurs de la General Electric $C^{\circ}$, qui produisent du courant triphasé à 6600 volts, dont la tension est élevée à 33000 volts au moyen de transformateurs $\left(^{\star}\right)$. A la pleine charge normale de $8000 \mathrm{HP}$, le débit de la conduite forcée est de $13 \mathrm{~m}^{3}$ à la seconde, correspondant à une vitesse de $2^{\mathrm{m}} 50$. Un troisième groupe de “óserve est actuellement on montage.

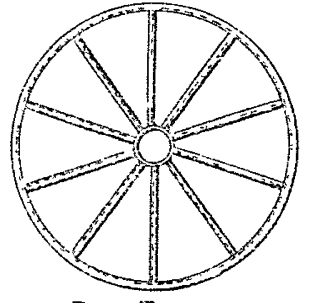

Roof Framino

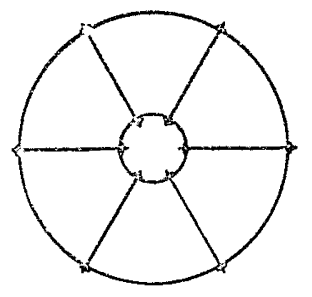

SECTIONA-A
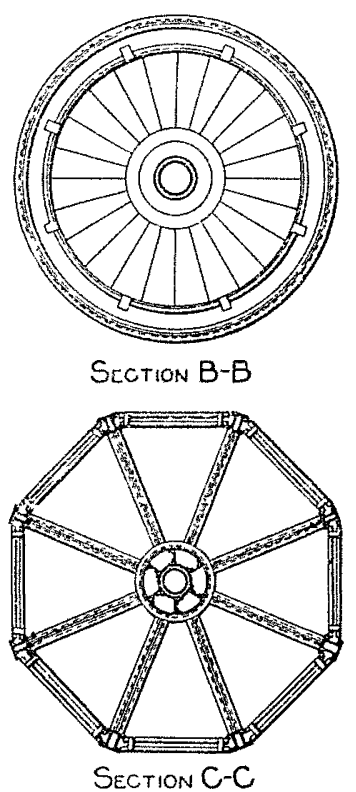

SECTION C-C

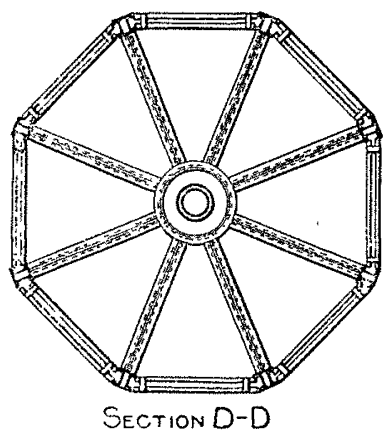

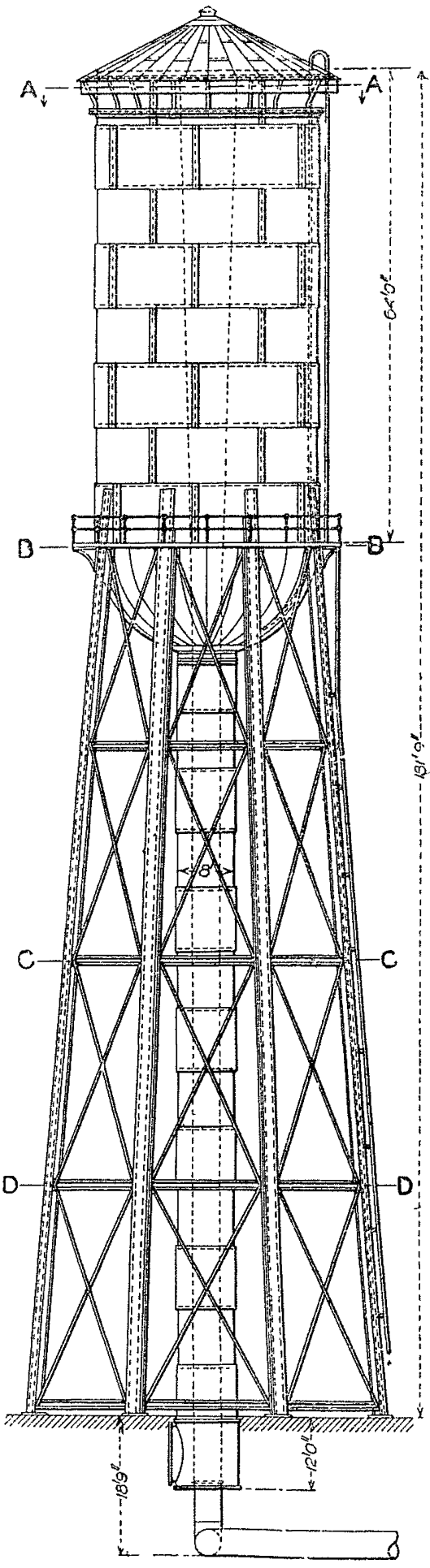
de coups de bélıer, et de la tour cua le supporte.

Lusine devant assurer un service de traction se trouve soumise à de brusques ct importantes variations de charge, ce qui entraîne de parellles variation dans la vitesse d'écou-

(·) Le tunnel dont il est question traverse la chaine des montagnes de li cilscade à $1000 \mathrm{~m}$ d'altıtude. Il a $4 \mathrm{~km}$ de longueur, el sa voie d'accès du côté est a une rampe de 20 pour 1000 . Il est situé à $50 \mathrm{~km}$. de l'usine. lemen $l$ de l'cau dans la conduite forcée. Afin de diminuer l'importance des coups de bélier, et de régulariser la pres. sion sur le distributeur des turbines, on a construit, tout à côté de l'usine, un róservoir spécial surélevé, de $1280 \mathrm{~m}^{3} \mathrm{de}$ capacité, qui est directement relić à la conduite forcée.

Ce réservoir est monté sur une tour en acier, et est cons. titué par un cylindre A B, de $9^{\mathrm{m}} \mathbf{4}$ de diamètre, et de $19^{\mathrm{m}} 50$ de hauteur au-dessus de la calotte hémisphérique qui le termine à sa partie inférieure. Le sommet de ce cylindre est à $55 \mathrm{~m}$. au-dessus du sol, au point où il est érigé, et à $3 \mathrm{~m}$. au-dessus de la crête du barrage de prise d'eau.

La tour qui supporte ce cylindre est formée par l'assemblagre de 8 pilier's, légèrement inclinés sur la verticale (1/12), de $36 \mathrm{~m}$. environ de hauteur. Chacue pilier est constitué par quatre tronçons de deux poutrelles en $\mathrm{U}$, de $9 \mathrm{~m}$. de longueur environ et de $38,1 \mathrm{~mm}$ de hauteur, qui sont assemblées, par leurs semelles extérieures, au moyen d'un plat de $50,8 \mathrm{~m} \mathrm{n}$. de larereur et de $18,7 \mathrm{~mm}$. d'épaisseur. Ces piliers sont cntretoisés vertıcalement et horizontalement comme lindique la figure ci-jointe. Les poutrelles du dernier tronçon sont taillées en biseau à leut partie supéricure, pour venir s'adapter contre les parois verticales de la partre inférieure du cylindre constituant le réservoir.

Suivant l'axe vertical de la tour, sont disposés concentriquement deux tuyaux, qui ont $2^{\text {m4 }} 4$ et $0^{\text {m99 }}$ de diamètre intérieur. Le tuyau extérieur est relié à sa partie in lérieure au collocteur des turbines; à son extrémité supérieure, il est fixé à la partie inférieure d'un joint de dilatation, en fonte, qui est muni d'un presse-étoupe. A sa partie supérieure, ce joint de dilatation est lui -même rivé à une couronne cylindrique qui termine la calotte hémisphérique du fond du réservoir. Ce tuyau extérieur est for'mé de tôles de 7,9 à 14,3 mm. d'épaisseur.

Le tuyau intérieur sert de trop-plein lorsque la cheminée vient à cracher sous l'effet d'un coup de bélier. A cet effet, son diamètre extérieur, qui est de $1{ }^{\mathrm{m}} 09$ sur la hauteur de la tour, va en croissant à partir du fond de la calotte hémisphérique jusqu'à $0^{m} 60$ en dessous du sommet du cylindre, où il atteint $2^{\mathrm{m}} 44$, de manière à former déversoir de trop-plein. Comme ce tuyau intérieur est soumis à un effort de compression, on lui a donné une épaisseur de $10 \mathrm{~cm}$. environ. Il est constitué par deux tôles d'acier concentriques, espacées de 7,62 cm., entre lesquelles on a coulé du béton. L'épaisseur de la tôle intérieure est uniformément de $9,52 \mathrm{~mm}$., tandis que celle de la tôle extérieure varie de 9,52 à 19,04 millimètres.

Les tôles d'acier qui constituent la calotte hémisphérique formant le fond du réservoir ont $14,3 \mathrm{~mm}$. d'épaisseur. Celles qui constituent les parois verticales du cylindre ont une épaisseur qui varie de 19,04 $\mathrm{mm}$. à la base, à $9,52 \mathrm{~mm}$. au sommet.

Le réservoir est couvert par un toit conique, de $10^{\mathrm{m} 97}$ de diamètre à la base, et de $2^{\mathrm{m}} \mathbf{7} 4$ de hauteur. On y accède au moyen d'une échelle métallique, visible sur la figure contre la paroi droite du cylindre, qui aboulit à une passerelle circulaire ménagée à la base du cylindre. On arrive à cette passerclle au moyen d'un escalier accolé contre le pilicr de droite de la figure ci-jointe.

Remarque.- Il existe déjà plusieurs installations hydroćlectriques où l'on a fait emploi de cheminées de sûreté, mais ces dispositifs ne se composaient simplement que l'un tube d'équilibre, de scction uniforme, sans réservoir de grande capacité.

Lorsque la section $\sigma$ du tube d'équilibre est du même ordre de grandeur que celle $S$ de la conduite forcée qu'il doit proteger, le coup de bélier positif maximum, ou de compression, lors d'une fermeture brusque, n'est plus qu'une fraction $\frac{S}{S+\sigma}$ du coup de bélier ordinäire.

Sur la plus grande partie de la cheminée de lusine de Tumwater Canyon, la section du tube d'équilibre est celle 
d'un tuyau creux, ayant $2^{\mathrm{m}} 4.4$ et $1^{\mathrm{m} 09}$ de diamètre. Dans ce cas, la fraction précitée est approximativement:

$$
\frac{S}{S+\sigma}=\frac{2,59^{2}}{2,59^{2}+2,44^{2}-1,09^{2}}=\frac{3}{5}
$$

En portant à $2^{m} 86$ le diamètre extéricur du tube d'équilihre, le coup de bélier positif maximum n'aurait été que la moitié du coup de bélıer sans cheminée d'équılibre.

Le réservoir a surtout pour but de parer aux coups de bélier négatifs. On sait, en eflet, que, au moment de l'ouverture brusque du distributeur, il se produit une dépression, qui est d'autant plus forte que l'ouverture a été plus rapide, ct que la conduite d'alimentation est plus longue, et cette dépression peut d'ailleur's être suivie d'une surpression. Avec la cheminée d'équilibre, la longueur qui intervient pour la formation de ce coup de bélier est approximativement celle de la cheminée, à la condition toutelois que cette cheminée soit capable de fournir assez d'eau pendant que le régime s'établit dans la conduite forcée d'alimentation. C'est pour cela qu'il est nécessaire d'avoir un réservoir de grande dimension. Toutefois, il semble, à premièro vue, que la capacité du réservoir de Tumwater Canyon aurait pu être un peu moindre.

L'Engineering Record, auquel les renseignements donnés sur ce réservoir sont empruntés, n’indique pas les bases qui ont servi au calcul des dimensions du réservoir. Il se contente de dire que, " par suite des variations brusques et considérables de la charge de l'usine, il faut environ 2 minutes pour vaincre l'inertie, et produire l'accélération maxima de la vitesse de l'eau dans la conduite forcée ». Ce temps nous paraît exagéré, car la phase du coup de bélier direct n'est ici que de 7 secondes $\left({ }^{\star}\right)$.

Avant de terminer, faisons remarquer que les déchargeur's qu'on installe souvent à côté des turbines, et qui provoquent l'écoulement d'une certaine quantité d'eau, lors d'une fermeture brusque du distributeur, n'ont qu'un effet d'amortissement des coups de bélier positifs; ils sont sans effet contre les coups de bélier négatifs. Or, si ceux-ci ne sont pas dangereux pour les conduites forcées, ils influent d'une manière défavorable sur le réglage des groupes électrogènes.

H. B.

\section{LE MOIS HYDRO-ÉLECTRIQUE}

\section{ACADÉMIE DES SCIENCES}

\section{MÉGANIQUE ET ÉLEGTRIGITÉ}

Nouvelles observations sur les courants telluriques entre stations à grande différence d'altitude. - Note de MM. B. Brunhes et P. David. Séance du 5 juillet rgog.

Dans une précédente communication $\left({ }^{\star *}\right)$, nous avons signalé nos études, poursuivies depuis 1904, sur les courants telluriques dans une ligne télegraphique allant de la faculté des Sciences de Clermont au sommet du Puy-de-Dôme, et nous avons appelé l'attention sur un double caractère de ces courants: y $^{\circ}$ grande différence de potentiel normale entre stations extrêmes; $2^{0}$ valeur exceptionnellement élevée des perturbations par temps de trouble magnétique. Ces deux propriétés semblaient, à priori, corrélatıves. Notis avons reconnu, au contraire, qu'elles tzennent à des portions différentes de la ligne.

Nous avons établi deux prises de terre intermédiaires, l'une à la station de la Font-de-l'Arbre, l'autre au pied même du Puy-de Dôme,

\footnotetext{
${ }^{*}$ ) On appelle phase du coup de bélier direct, le rapport $\frac{2 l}{a}$ de la longueur $l$ de la condute forcée à la vitesse $a$ de propagation des presslons variables, $a$ vaut approximativement $1000 \mathrm{~m}$. à la seconde or $l=3600 \mathrm{~m}$., d'où une période d'environ 7,2 secondes pour la phase du coup de bülier drect.

(") Comptes Rendus, 2 I décémbre 19o8.- Voir aussi La Houlle Blanche de mars I 909 .
}

dans la plaine de Laschamps, et mesuré la différence ce potentiel moyenne sur chacun des tro1s tronçons ainsi constitués. Le tableau ci-joint définit la position des prises de terre, et donne les différences de potentiel $\left({ }^{*}\right)$.

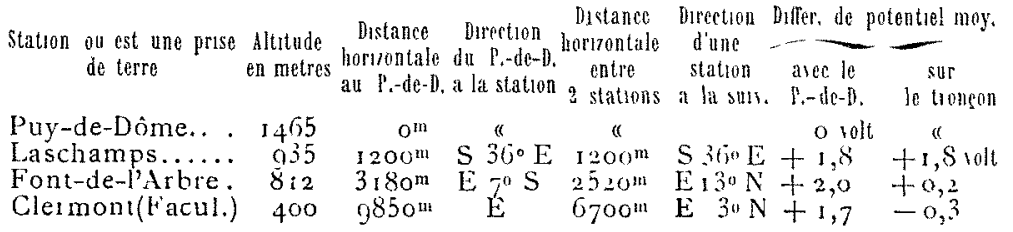

Le potentiel, à chaque station, a une très faible varıtion diurne par calme magnétique. Mais"1l y a une varıation annuelle qu'll y aura lieu de preciser et qui arrive, partors, à rendre incertan le sens du courant pris entre Laschamps et la Faculte. En tous les cas, le potentiel passe par un maximum à la Font-de-l'Arbre, et la grosse difference de potentiel normale existe entre le sommet et le pted de la montagne.

Pour localiser les différences de potentiel occasionnelles provenant des orages magnétiques, nous avons enregistre, $\dot{a}$ la fols, pendant un certain nombre de nuits, en mai et juin 1909 , le courant tellurique sur le tronçon du Puy-de-Dôme à Laschamps (à l'arde du millrampèremètre enregistreur de la station du sommet), et le courant tellurique sur le tronçon de la Font-de-l'Arbre à la Faculté (à l'aide d'un galvanomètre shunté et de l'enregistrement photographique). (1) a d'alleurs pris la précaution d'enregistrer souvent le courant sur la ligne entière avec les deux enregistreurs de type différent, en série aux deux bouts, et de vérifier l'identité des courbes obtenues. De part et d'autre, on graduait les courbes en insérant dans la ligne, durant 5 ou ro minutes, un accumulateur chargé, ce qui donnait directement, et indépendamment de la résistance des prises de terre, la valeur de l'ordonnée en volts.

Prenons pour exemple la nuit du ir au 19 mai igog. Le i 8 , cntre $2 \mathrm{I} \mathrm{h} .20 \mathrm{~m}$. et $2 \mathrm{I}$ h. 28 , la différence de potentiel, sur la ligne de la Font-de.l'Arbre à la Faculté, a varié de 1,875 volts, soit 280 millivolts par kilomètre. Au même moment, le courant sur la ligne de Laschamps au Puy-de-Dôme a subı une variation correspondant à moins de 0,12 volt, soit moins de 100 millivolts par kilomètre.

Les courbes ont été comparées à celles de Tortosa. Le courant Est-Ouest de Toitosa a donne une courbe semblable à la nôtre, mais avec une amplitude de perturbations beaucoup plus fable. La perturbation de $2 \mathrm{I} \mathrm{h} .28 \mathrm{~m}$. correspond seulement à 14 millivolts par kilomètre. Par contre, le courant Nord.Sud de Tortosa a présenté, la nuit du 18 au 19 , des perturbations atteignant 300 millivelts par kilomètre.

La comparaison d'autres périodes troublées a donné des résultats du même ordre.

Le rappro:hement des courbes de Tortosa et des nôtres permettrait de conclure à une absence de proportionalité entre les composantes perpendiculaires des perturbations aux deux observatoires, c'est-à-dire à une différence dans la dırection des courants telluriques, si notre - tronçon Nord-Sud (ou au moins dont la composante principale est Nord'Sud) n'était pas en même temns très inclinée par rapport à l'horizon: il faudrait avoir une ligne Nord-Sud en plaine, ou à peu près, ce qui nous manque.

Mais une double conclusion s'impose dès mantenant : La sensibilité de notre ligne Est-Ouest aux perturbations magnétıques, sensitilité de l'ordre de vingt fois celle de la ligne Est-Ouest de Tortosa, ne tient pas à l'énorme difference d'altitude entre le Puy-de-Dóme et Clermont. Il est donc raisonnable de penser qu'en d'autres stations on pourra établir des lignes télégraphiques courtes de sensibilite analogue.

Les lignes Est-Ouest sont caractérisées par la faiblesse de la variation diurne des courants télluriques qui les traversent en temps de calme. Tandis que dans les observatoiı es magnétiques d'Europe, en général, on caractérise comme journées calmes (o de la conlérence d'Innsbruck) les journées où les variations accidentelle's de la déclinaison, par exémple, n'ont pas atteint le tiers (ou, en certain cas, la moitié) de l'amplitude de la variation diurne du même élément, et comme journées très troublées ( 2 d'Innsbruck) celles où les varıtions accidentelles ont dépassé l'amplitude de la variation diurne, nous avons été conduits à noter du chiffre o les journées où les perturbations sur notre ligne Est-Ouest n'ont pas atteint 50 millivolts par kilomètre, et à réserver le degré 2 aux journées où les perturbations ont dépassé I 70 milivolts par kilomètres. Et la publıation trimestrielle du Caractère magnétique prouve que notre classement des jours du mois concorde aussi bien avec ceux que donnent les autres observatoires magnétıques, que ceux-ci entre eux.

La recherche des régions oú 11 serait possible davoir des lignes dırigées de l'Est à l'Ouest, à faible variatıon diurne, et à grande sensibilité aux perturbations, recherche qu'autorisent nos résultats, aurait, à côté d'un intérêt theorıque considérable, un intérêt pratique de premier ordre, en fournissant le moyen le plus simple dinscrire ¿es troubles magnétiques.

(*) Les directions indiquées sont celles des lignes droites allant d'une station, soit au Puy-de-Dóme, soit à la station survante. 